\author{
Cadernos de \\ ESTUDOS LINGḯlSTICOS - (57.1), Campinas, Jan./Jun. 2015
}

\title{
SOBRE A ESTRUTURA DAS SENTENÇAS PSEUDO-CLIVADAS E SEMI-CLIVADAS
}

\author{
CARLOS MIOTO \\ Universidade Federal Fronteira Sul/CNPq* \\ MARY A. KATO \\ Universidade Estadual de Campinas/CNPq
}

\begin{abstract}
RESUMO: O objetivo desse artigo é estudar as sentenças pseudo-clivadas (PCs) e semi-clivadas (SCs) e propor, para elas, uma estrutura diferenciada que atenda suas especificidades. A estrutura proposta para as PCs leva em conta o que muitas vezes é chamado de Efeitos de Conectividade. A estrutura proposta para as SCs leva em conta o fato de que apenas constituintes no domínio de c-comando de T podem ser focalizados. Descartando que PCs e SCs possam ter uma derivação comum, procuramos explicar as várias assimetrias entre elas observadas na literatura.
\end{abstract}

Palavras-chave: focalização; pseudo-clivadas; semi-clivadas.

\begin{abstract}
The aim of this article is to analyze pseudo-cleft (PC) and semi-cleft (SC) sentences, and contrary to tradition, propose to them different derivations that meet their specificities. The resulting structure proposed for the PCs take into account what has been known as its 'connectivity effects'. The structure proposed for the SCs take into account the fact that, contrary to PCs, only constituents in the c-command domain of $\mathrm{T}$ can be focalized. Abandoning a common derivation for PCs and SCs, we succeed to explain other asymmetries between them.
\end{abstract}

Keywords: focalization; pseudo-clefts; semi-clefts.

\section{INTRODUÇÃO}

O objetivo desse trabalho é analisar os dois tipos de construções exemplificados em (1):

(1) a. O que a Maria é é escandalosa.

b. A Maria é é escandalosa.

* $\mathrm{O} C N P q$ deu suporte para a pesquisa que resultou nesse trabalho ao primeiro autor processo $\mathrm{n}^{\circ}$ 308351/2011-8 e à segunda processo $\mathrm{n}^{\mathrm{o}}$ 305515-2011-0.

Esse trabalho foi desenvolvido no seio do projeto Romania Nova concebido para incentivar estudos envolvendo línguas da România Velha faladas, em especial, na América Latina e implantado por Mary A. Kato. Presto minha homenagem à Mary, que enriqueceu meu trabalho tornando-se desde muito cedo minha parceira. 
Nas duas sentenças de (1) escandalosa é lido como o foco. (1a) é reconhecido tradicionalmente como uma pseudo-clivada (PC) (Akmajian 1970). E em virtude de (1b) diferir de (1a) apenas pela falta da expressão WH o que, ela é muitas vezes chamada de semi-clivada (SC) (Kato 2010).

As PCs são encontradas irrestritamente no português e no espanhol (exemplos em (2) de Camacho 2006).

(2) a. Lo que le gusta es bailar salsa.

o que lhe agrada é dançar salsa

b. Le gusta es bailar salsa.

Porém, enquanto as SCs são comuns no português, no espanhol elas se restringem ao que é falado na região do Caribe (Bosque 1999)1.

As primeiras análises das SCs investem na semelhança linear: Wheeler 1982 postula que em (1b) o operador WH presente em (1a) foi apagado, seguindo Chomsky 1977; Toríbio 1992 postula que (2b) contém um operador WH nulo, seguindo Chomsky 1981. Para elas, a diferença entre PCs e SCs se restringe à presença ou ausência da expressão $\mathrm{WH}^{2}$.

Pretendemos nesse artigo defender, ao contrário de Wheeler 1982, Toríbio 1992 e Costa e Duarte 2003, que PCs e SCs não têm derivações comuns.

\section{PCS}

Akmajian 1970 aponta que uma sequência como (3) pode ter leitura predicacional (4a) ou especificacional (4b) (ver Higgins 1973 para uma discussão detalhada dessa ambiguidade).

(3) What Mary is is scandalous

(4) a. O que a Maria é é escandaloso.

b. O que a Maria é é escandalosa.

Ao traduzirmos (3) para o português, não há como preservarmos a ambiguidade já que, se é do tipo biforme, o adjetivo tem que concordar com o que é seu argumento. Isto é, a forma não marcada escandaloso denuncia por concordância que o argumento do adjetivo só pode ser a relativa livre [o que a Maria é ] em (4a), enquanto a forma marcada escandalosa mostra que o argumento só pode ser a Maria em (4b).

\footnotetext{
${ }^{1}$ De acordo com o autor, esta diferença "pode ser um caso de influência de substrato de uma ou várias línguas do Oeste da África, pois muitas delas têm marcadores morfológicos para constituintes focalizados". (p.26)

${ }^{2}$ Costa e Duarte (2003) também analisam as semi-clivadas como contendo um operador WH nulo no português europeu.
} 
Cadernos de ESTUDOS LINGǘISTICOS (57.1) - Jan./Jun. 2015

Para efeitos de focalização, há uma diferença fundamental entre (4a) e (4b): na primeira, tanto a relativa livre [o que a Maria é] quanto o adjetivo escandaloso podem ser focalizados, como mostram os diálogos indutores do foco, que está grifado em (5).

(5) a. - O que é escandaloso?

- $\underline{\mathrm{O} \text { que a Maria é é escandaloso. (Escandaloso é o que a Maria é.) }}$

b. - O que a Maria é é o quê?

- O que a Maria é é escandaloso.

Entretanto, em (4b) somente o adjetivo escandalosa pode ser o foco (mesmo que se altere a ordem dos constituintes). Se admitimos que uma PC é uma sentença designada para focalizar um constituinte, exatamente aquele que aparece depois da (segunda) cópula na versão canônica, apenas (4b) deve ser considerada uma PC. Por sua vez, a sequência em (4a) não deve ser considerada uma PC, mas uma sentença predicativa ordinária. $\mathrm{O}$ aparato formal usado para configurar uma $\mathrm{PC}$ se compõe de uma a expressão WH formadora do constituinte que imita uma relativa livre e da cópula que isola o foco.

\section{CONTRA UMA DERIVAÇÃO COMUM PCS E SCS}

Definido que uma PC é, no sentido relevante, necessariamente especificacional, temos a percepção do motivo por que muitas vezes SCs e PCs recebem um tratamento semelhante, como fazem Wheeler 1982, Toríbio 1992, Costa e Duarte 2003. Compare (4) com (6):

(6) a. A Maria é é escandalosa.

b. *A Maria é é escandaloso.

Se entendemos que, de alguma forma, uma SC é uma PC sem a expressão WH e que uma sentença predicativa não é uma $\mathrm{PC}$, entendemos imediatamente por que, retirando a expressão WH, apenas (6a) é gramatical. Além disso, o fato de que PCs e SCs são sentenças designadas para focalizar constituintes parece corroborar a unificação de tratamento e uma derivação comum para os dois tipos de sentenças.

Uma observação mais cuidadosa de (1) e (2) mostra, entretanto, que há entre elas assimetrias relevantes que colocam em cheque a hipótese de que a diferença se limita ao que é visível: a presença ou ausência da expressão WH.

Bosque 1999 foi o primeiro a apontar assimetrias entre PCs e SCs a partir do espanhol falado no Caribe:

As SCs são incapazes de focalizar um constituinte deslocado, diferente do que acontece com as PCs: 
(7) a. Um empleo es lo que Juan necesita

b. *Um empleo es Ø Juan necesita

De modo semelhante, uma expressão WH interrogativa pode ser movida para Spec de CP numa $\mathrm{PC}$, mas não pode numa SC:

(9) a. ¿Qué era lo que Juan leía?

b. *¿Qué era Juan leía?

Nas PCs é necessário o verbo proforma hacer para focalizar o VP, diferente do que se observa nas SCs:

(8) a. Lo que Juan hizo fue cantar (não causativo)

b. *Juan hizo fue cantar

Quando anteposto ao verbo da relativa livre, o advérbio más força a interpretação superlativa para as PCs, mas não para a SCs:

(10) a. Lo que más le gusta es bailar salsa

b. Más le gusta es bailar salsa

Advérbios focalizadores como solo têm escopo diferente se antecedem o verbo lexical numa PC e numa SC:

(11) a. Lo que solo leí fue este libro (só li, *só este livro)

b. Solo leí fue este libro (só li ou só este livro)

Das três primeiras assimetrias, que são de ordem estrutural, a primeira e a segunda se estendem diretamente ao português. A que se refere ao proforma fazer deve ser qualificada: para focalizar o VP, o proverbo fazer é necessário nas PCs, mas sujeito à variação dialetal nas SCs:

(12) A falecida nossa mãe só fez foi esperar (pela escritura das terras) (FSP, 20/11/2010, reproduzindo a fala de um quilombola do sul de São Paulo)

As duas últimas são de caráter semântico e parecem se aplicar ao português. Mioto $(2008,2012)$ também aponta algumas assimetrias entre PCs e SCs observadas a partir do português brasileiro (PB):

As SCs não são talhadas para expressar foco de informação, como vemos na inadequação (\#) da resposta (13c) a (13a); por outro lado, embora redundante, (13b) responde adequadamente à pergunta (13a):

(13) a. - O que a Maria bebeu?

b. - O que a Maria bebeu foi cachaça.

c. \#-A Maria bebeu foi cachaça. 
Quando se trata de focalizar o sujeito, duas situações se apresentam de acordo com o fato de o sujeito ser de terceira pessoa do singular ou não. Não sendo o sujeito de terceira pessoa do singular, a assimetria entre PCs e SCs é categórica, pelo menos no PB:

(14) a. Quem fez a trapalhada fui eu.

b. ?*Fez a trapalhada foi/fui eu.

c. ?Fiz a trapalhada fui $\mathrm{eu}^{3}$.

A presença do quem como sujeito de fazer em (14a) leva o verbo para a terceira pessoa nas PCs enquanto a cópula concorda com o eu posposto. $\mathrm{Na}$ ausência de quem, se fazer fica na terceira pessoa como em (14b), vamos ter um desacordo com o sujeito eu; se os dois verbos concordam na primeira pessoa como em (14c), não se pode postular um operador vazio como sujeito do verbo fazer. Quando o sujeito é de terceira pessoa, as SCs apresentam variação dialetal enquanto os julgamentos das PCs não variam:

(15) a. Quem fez a trapalhada foi o João.

b. *Fez a trapalhada foi o João.

(apud Mioto 2008)

(16) a. Quem me ajudou muito foi o psicólogo.

b. Me ajudou muito foi o psicólogo.

(apud Wheeler 1982)

A mesma variação em relação às SCs parece ser encontrada no espanhol caribenho, como se deduz de (16):

(16) a. Compró los libros fue Pedro

(apud Camacho, 2006)

b. *Ganó el premio fue [el estudiante cubano]. (apud Toribio 1992)

3) Quando se trata de focalizar o VP de uma perífrase verbal, uma SC o faz naturalmente enquanto uma PC resulta agramatical:

(17) a. *O que o João vai é viajar.

b. O João vai é viajar.

(18) a. *O que o Lula tem é falado pouco e com poucos.

b. O Lula tem é falado pouco e com poucos.

(FSP, 31/10/2006)

Para focalizar um VP com uma PC é necessário recorrer ao proforma fazer, como em (19):

${ }^{3}$ Essa sentença nos parece funcionalmente estranha porque o que é focalizado pela cópula é denunciado antes pela presença da concordância de primeira pessoa. Veja que isso não acontece nas PCs uma vez que o quem esconde qual é o foco. 
(19) a. O que o João vai fazer é viajar.

b. O que o Lula tem feito é falado pouco e com poucos.

4) Outro fenômeno que reforça a assimetria entre PCs e SCs é a Concordância Negativa. Em (20a) o não antes do verbo respeitar licencia naturalmente ninguém após a cópula. Em (20b), porém, isso não acontece e o que temos é uma sentença degradada:

(20) a. O João não respeita é ninguém.

b. ??Quem o João não respeita é ninguém.

Em resumo, as assimetrias apontadas por Bosque 1999 e Mioto 2008, 2012 sugerem fortemente que SC não deve ser encarada como uma PC à qual falta uma expressão WH pronunciada. Sugerem, também, que os dois tipos de sentença não devem ter uma derivação comum.

\section{PCS}

\subsection{Análises prévias}

Wheeler 1982 propõe que uma PC é formada por uma relativa livre (RL) sujeito seguida de um predicado:

(21) a. $\left[_{R L} \mathrm{O}\right.$ que quero] [ ${ }_{\text {predicado }}$ é que você vá pra casa]

b. ${ }_{\mathrm{RL}}$ Do que estou farta] [predicado $_{\text {é dessa palhaçada }]}$

Admitindo que PCs são sentenças copulares linguistas portugueses e brasileiros propõem que elas derivam de uma cópula que requer uma mini-oração como complemento. Modesto 1995 e Kato et alii 1996 postulam que o foco é o sujeito da mini-oração e a RL é o predicado (22b); e Costa e Duarte 2003 que a RL é o sujeito e o constituinte focalizado é o predicado (22c):

(22) a. Quem eu vi foi a Maria.

b. $\left[_{\text {IP }}\right.$ Expl foi $\left[_{S C}\left[{ }_{\text {sujeito }}\right.\right.$ a Maria $]\left[_{\text {predicado }}\right.$ quem eu vi $\left.\left.]\right]\right]$

c. $\left[_{\mathrm{IP}}\right.$ foi $\left[_{\mathrm{SC}}\left[{ }_{\text {sujeito }}\right.\right.$ quem eu vi $]\left[_{\text {predicado }}\right.$ a Maria $\left.\left.]\right]\right]$

A variação na ordem dos constituintes depende de qual deles é alçado.

As propostas resumidas em (22) parecem derivar diretamente a versão predicacional de uma sequência como (23):

(23) O que o João é é escandaloso.

Independente da ordem subjacente das propostas em (22), na versão predicacional [o que o João é] é uma RL que funciona como argumento do 
adjetivo escandaloso e como sujeito da sentença, de modo semelhante a (4a), aqui repetido:

(4) a. O que a Maria é é escandaloso.

b. O que a Maria é é escandalosa.

A concordância do adjetivo com a RL atesta isso. Entretanto, a concordância do adjetivo com a Maria em (4b) mostra crucialmente que as derivações em (22) não conseguem lidar confortavelmente com (4a).

$\mathrm{O}$ que parece ser claro é que os linguistas que trabalharam com o português estavam traçando a estrutura de uma sentença predicacional, sem terem em conta as complicações, que serão agrupadas abaixo sob o rótulo de Efeitos de Conectividade, envolvidas numa especificacional.

\subsection{Efeitos de Conectividade}

Alargaremos o conceito de Efeitos de Conectividade (Akmajian 1970, Higgins 1973) de modo a abrigar vários fenômenos exemplificados abaixo (exemplos (24) a (27) retirados de Boskovic, 1997):

(24) Relação entre antecedente e anáfora

What John is is important to himself.

(25) Possessivo ligado por quantificador

What everyone proved was his own theory.

(26) Licenciamento de Itens de Polaridade Negativa ${ }^{4}$

What John didn't buy was any picture of Fred.

(27) Caso acusativo

Was Hans essen wollte war einen Apfel.

$\mathrm{O}$ que $\mathrm{H}$. comer queria era uma ${ }_{\mathrm{Acc}}$ maçã

(28) Concordância do adjetivo e marcação temática

O que a Maria é é escandalosa.

Observemos, em primeiro lugar, que nenhuma das sentenças de (24) a (28) é ambígua: a única interpretação disponível para cada uma delas é a especificacional. Ademais, em cada uma das sentenças (24) a (28), falha a condição que regula a relação entre os itens em negrito e não se vislumbra como a falha pode ser anulada por reconstrução. Em (24) John não c-comanda himself; em (25) everyone não c-comanda his; em (26) n't não c-comanda any. Em (27) não há localidade entre o verbo essen (comer) e o DP einem Apfel (uma maçã) necessária para a atribuição

\footnotetext{
${ }^{4}$ Sabemos que em português as condições que regulam o licenciamento dos Itens de Polaridade Negativa não são as mesmas que atuam no inglês. A aceitabilidade da tradução da sentença em (26) para o português é duvidosa:

(i) ?O que o João não comprou foi nenhuma pintura do Fred.
} 
(checagem) do caso acusativo. Em (28) não se verifica a relação Spec-núcleo que regula a concordância de escandalosa com a Maria, nem a condição de localidade que regula a marcação temática do DP a Maria.

Mioto e Negrão 2007 e Resenes 2009 põem em dúvida que a construção WH na sentença especificacional seja uma verdadeira RL em vista de dois fenômenos principais: o primeiro é que, na esteira de Hankamer 1974, ela não pode sofrer movimento A, como vemos em (29b); o segundo é, na esteira de Ambar (2001), que RLs nunca exigem concordância temporal entre o verbo interno à construção WH e a cópula, necessária em (30b), mas não em (30a).

(29) a. [O que a Maria é $]_{i}$ parece $t_{i}$ ser escandaloso

b. *[O que a Maria é $]_{\mathrm{i}}$ parece $\mathrm{t}_{\mathrm{i}}$ ser escandalosa

(30) a. [O que a Maria é] (já) foi escandaloso

b. *[O que a Maria é] (já) foi escandalosa

Além disso, quando conseguimos construir pares mínimos com sentenças predicacionais e especificacionais vemos que a situação muda nos aspectos relevantes. Em (31) o sintagma ein Apfel (uma maçã) é marcado por nominativo, trivialmente o caso do predicativo do sujeito; em (32) o sintagma a Maria não é capaz de licenciar a anáfora dentro do foco.

(31) Caso

Was Hans essen wollte war ein Apfel. (cf. Boskovic 1997)

$\mathrm{O}$ que H. comer queria era uma ${ }_{\text {Nom }}$ maçã

(32) Relação entre antecedente e anáfora

*O que a Maria é é digno de si mesma. (Sérgio Menuzzi, c.p.)

Em suma, a estruturação interna à construção WH de uma PC genuína, apesar de criar um ambiente sintático aparentemente desfavorável, não impede que se estabeleça a conexão entre os itens relevantes em (24) a (28). Por outro lado, quando temos uma verdadeira RL, a conexão é impedida.

\subsection{Uma análise derivacional ${ }^{5}$ : Boeckx $2007^{2}$}

Boeckx 2007 centra sua análise das PCs em três pontos: a natureza de what, o caráter de foco do contrapeso e a natureza e as restrições de tempo da cópula. Os estágios relevantes da derivação de uma PC são reproduzidos em (33):

${ }^{5}$ Uma análise que leva em conta os Efeitos de Conectividade foi desenvolvida por Boskovic 1997. A proposta procura explorar a ideia semelhante à nossa de que a cópula e a expressão WH usados para montar uma PC são itens puramente formais que não impedem que a conexão requerida se estabeleça. A expressão WH, que é um operador nas RLs, é considerada uma variável nas PCs. A proposta de Boskovic 1997 procura reconstruir as estrutura em LF de tal modo que a condição de c-comando seja apropriadamente reestabelecida. Por não ser propriamente derivacional, ela não será abordada detalhadamente aqui. 
(33) a. John is important

b. [FocP important [IP [VP John is t]]]

c. [TopP [John is $\left.t_{i}\right]_{j}$ [FocP important ${ }_{i}$ [IP [VP $\left.\left.\left.\left.t_{j}\right]\right]\right]\right]$

d. [TopP [What $t_{i} J_{\text {ohn }}$ is $\left.t_{i}\right]_{j}$ [FocP important [IP [VP $t_{j}$ ]]]]

e. [TopP [What ${ }_{i} J_{\text {ohn }}$ is $t_{i}$ ] is [FocP important [IP [VP $\left.t_{j}\right]$ ]]

A sentença simples em (33a) é derivada trivialmente. Para passar a (33b) important é movido para FocP para checar o traço de foco. Para passar a (33c) o VP [John is $t_{i}$ ] é movido para TopP para checar o traço de tópico. O toque para passar a (33d) consiste em um processo que revive important como o resumptivo what em Spec de TopP para criar a estrutura predicativa presente na relativização. Para chegar a (33e) a cópula é inserida em Top ${ }^{\circ}$ criando a predicação alta, típica das estruturas de tópico-comentário.

A inserção da cópula em Top $^{\circ}$ postulada em (33e) é permitida em virtude do caráter puramente funcional desse item. Ela é diferente daquela que media a predicação baixa na área do IP (ver também Boscovic 1997). Por ser assim, ela não pode ter tempo independente do da sentença WH e deve participar de um processo de concordância temporal. Estando em Top ${ }^{\circ}$, a cópula está muito alta para participar de operações que, como I-to-C, dependem da existência de Infl.

\subsection{Uma proposta derivacional alternativa}

Vamos assumir que a expressão WH não tem o estatuto de operador, como nas RLs, mas funciona como uma variável cujo valor é atribuído pelo foco (cf. Boskovic 1977). Seguindo Kayne 2002 e outros, Kato e Mioto 2011 consideram que as relações interpretativas são derivadas por movimento sintático. A teoria de Kayne é exemplificada pelo fenômeno conhecido com Clitic Doubling do espanhol onde o antecedente e o associado aparecem combinados em um grande DP (35a):

(34) Le doy un libro a Juan.

(35) a. Doy un libro $\left[_{\mathrm{DP}}\right.$ Juan le]

b. [Juan le $]_{\mathrm{i}}$ doy un libro $\mathrm{t}_{\mathrm{i}}$

c. Juan ${ }_{j}\left[t_{j} l e\right]_{i}$ doy un libro $t_{i}$

d. a $\operatorname{Juan}_{\mathrm{j}}\left[\mathrm{t}_{\mathrm{j}} \mathrm{le}\right]_{\mathrm{i}}$ doy un libro $\mathrm{t}_{\mathrm{i}}$

e. $\left[\left[t_{j} l e\right]_{i} \text { doy un libro } t_{i}\right]_{k}$ a Juan $t_{k}$

A derivação da sentença em (34) é como em (35) mediante movimentos sucessivos e a inserção da preposição em (35d).

Baseados no caráter resumptivo da expressão $\mathrm{WH}$, postulamos que ela é combinada com o foco num grande constituinte, como fazemos em (37a):

(36) O que a Maria é é escandalosa. 
(37) a. A Maria é [o que [escandalosa]]

Movimento WH

b. $\left[{ }_{\mathrm{CP}} \mathrm{O}\right.$ que a Maria é $\left[\mathrm{t}_{\text {o que }}\right.$ [escandalosa $\left.]\right]$

Combinação da cópula

c. $\left[{ }_{\mathrm{VP}} \operatorname{ser}\left[{ }_{\mathrm{CP}}\right.\right.$ o que a Maria é [ $\mathrm{t}_{\mathrm{oque}}$ [escandalosa $\left.\left.\left.]\right]\right]\right]$

Movimento de escandalosa para a posição baixa de foco (cf. Belletti's 2004)

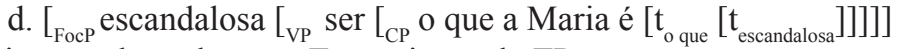

Movimento do verbo para T e projeção do TP

e. $\left[_{\mathrm{TP}}\right.$ é ${ }_{\mathrm{Focp}}$ escandalosa $\left[_{\mathrm{VP}} \mathrm{t}_{\text {ser }}\left[\mathrm{CP}\right.\right.$ o que a Maria é $\left.\left.\left.\left[\mathrm{t}_{\mathrm{o} \text { que }}\left[\mathrm{t}_{\text {escandalosa }}\right]\right]\right]\right]\right]$

Movimento do CP remanescente para TopP (como um bloco pressuposicional)

f. $\left[_{\text {TopP }}\right.$ o que a Maria é $]\left[_{T P}\right.$ é $\left[_{\text {FocP }}\right.$ escandalosa $\left.\left.\left.\left[_{\mathrm{VP}} \mathrm{t}_{\mathrm{ser}}\left[\mathrm{t}_{\mathrm{CP}}\right]\right]\right]\right]\right]$ presuposição foco

O passo (37f) da derivação é condizente com o fato apontado de que a construção WH não é realmente uma RL (cf. Resenes 2009) e como tal não pode sofrer movimento do tipo A ou A'. Mas, pode ser movida como um bloco por movimento remanescente criando uma estrutura de predicação (cf. Cf. Resenes e Den Dikken 2012, Resenes 2014) só que mais alta: estrutura típica de tópicocomentário.

Observe, ainda, que o passo que abriga a expressão WH sob um constituinte maior (cf. (37a)) resgata todas as condições, aparentemente violadas nas PCs, que permitem estabelecer as várias conexões:

(38) Relação entre antecedente e anáfora

John is [WH [important to himself]]

(39) Possessivo ligado por quantificador

Everyone proved [WH [his own theory]]

(40) Licenciamento de Itens de Polaridade Negativa

John didn't buy [WH [ any picture of Fred]]

(41) Caso acusativo

Hans essen wollte [WH [einen Apfel]]

$\mathrm{O}$ que $\mathrm{H}$. comer queria era uma Acc $_{\text {maçã }}$

(42) Concordância do adjetivo e marcação temática

A Maria é [WH [escandalosa]]

Entretanto, a derivação em (37) apresenta um problema semelhante ao da análise de Boeckx porque ela não exclui uma PC como (17a), aqui repetido, cuja derivação seria como (43):

(17) a. *O que a Maria vai é viajar. 
(43) a. A Maria vai [o que [viajar]]

b. [O que [a Maria vai [ $\mathrm{t}_{\text {oque }}[$ viajar $\left.\left.]\right]\right]$

c. [ser [o que [a Maria vai [ $\mathrm{t}_{\theta \text { que }}$ [viajar]]]]]

d. [viajar [ser [o que [a Maria vai [ $\left.\left.\left.\left.\mathrm{t}_{\text {oqute }}\left[\mathrm{t}_{\text {viajar }}\right]\right]\right]\right]\right]$

e. é [viajar [ $\mathrm{t}_{\text {ser }}$ [o que [a Maria vai $\left.\left.\left.\left[\mathrm{t}_{\text {oque }}\left[\mathrm{t}_{\text {viajar }}\right]\right]\right]\right]\right]$

f. [O que [a Maria vai $\left.\left.\left[\mathrm{t}_{\text {oque }}\left[\mathrm{t}_{\text {triajar }}\right]\right]\right]\right]$ é $\left[\operatorname{viajar}\left[\mathrm{t}_{\text {sef }}\right]\right]$

Nossa explicação sobre a razão por que (17a) é agramatical reside no fato de que, sendo uma variável, a expressão WH tem que ter uma função gramatical (nos termos da GB, precisa se submeter ao ECP). Isso não acontece dentro da construção WH em (17a), já que vai (o futuro não-afixal) é uma categoria funcional incapaz de lhe atribuir tal função à expressão WH. Essa incapacidade acontecerá sempre que a expressão WH redobra um VP complemento de T ou de qualquer verbo auxiliar'. Como mostra (19a), a inserção do proforma fazer 'salva' a sentença já que atribui função gramatical (objeto de) à variável:

(19) a. O que a Maria vai fazer é viajar.

A estrutura complexa de Kato e Mioto 2011 espelha um tipo de small clause equativa (cf. Den Dikken 2006). Na maioria das vezes, a estrutura complexa exibe efeitos de compatibilidade entre WH e XP:

(44) a. Com quem eu conversei foi com a Maria.

b. *Quem eu conversei foi com a Maria.

Entretanto, quando a preposição expressa caso inerente (cf. Kato 2009), ela é opcional estando em posição A', como em (45):

(45) (D)o que eu gosto é de manga.

\section{A ESTRUTURA DAS SCS}

\subsection{Análises prévias}

Mostrando que uma SC não pode ser uma PC sem a expressão WH nem com um WH nulo, Bosque 1999 propõe que ser não é um verbo copulativo, mas um operador de foco designado para focalizar contrastivamente o constituinte que o segue. O constituinte focalizado se situa necessariamente no domínio do VP. Para Bosque, a estrutura de uma sentença como (46a) é (46b):

${ }^{6} \mathrm{O}$ complemento infinitivo de querer pode ser focalizado:

(i) O que a Maria quer é viajar.

Mas nesse caso, o verbo querer é lexical e seu complemento é um CP. 
(46) a. Juan comía era papas.

b. $\left[\operatorname{Juan}_{\mathrm{i}}\left[_{\mathrm{VP}} \mathrm{t}_{\mathrm{i}}\right.\right.$ [comia $\left[_{\mathrm{FP}}[\mathrm{F}\right.$ era $]$ papas $\left.\left.\left.]\right]\right]\right]$ (apud Bosque 1999)

O verbo ser é o núcleo de FP e focaliza seu complemento.

Camacho 2006 aponta que a proposta em (46b) enfrenta um problema, a saber, a proposta não bloqueia a derivação de uma pergunta $\mathrm{WH}$, como (47b), com movimento do complemento da cópula:

(47) a. Los pájaros se comieron fue las migas.

Os pássaros cl comeram foi as migalhas

b. ${ }^{*}$ ¿Qué ${ }_{i}$ se comieron los pájaros fue $t_{i}$ ?

Ele faz uma proposta alternativa concebendo que a cópula é o núcleo de um sintagma copular ( $\left.\mathrm{IP}_{\mathrm{COP}}\right)$ que deve ser adjunto das fases fortes: à fase do $\mathrm{CP}$, quando se trata das PCs, e à fase do VP, quando se trata das SCs. No que se refere às SCs, a estrutura seria como (48):

(48)

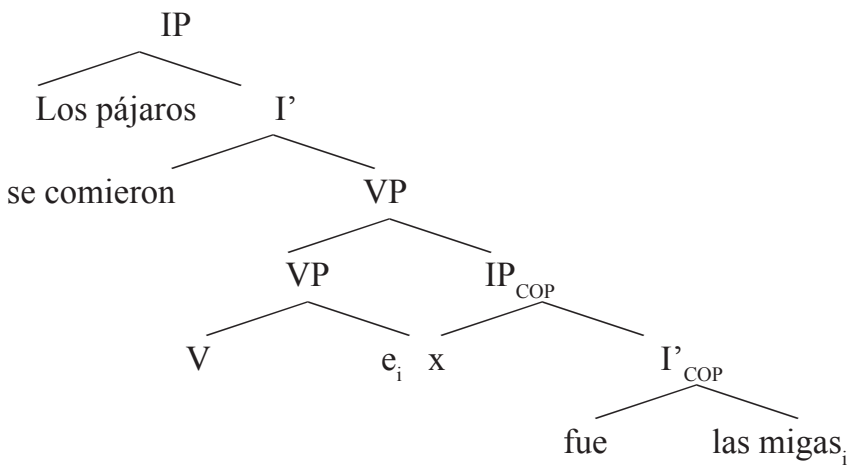

$\mathrm{IP}_{\mathrm{COP}}$ é uma estrutura completa com sujeito $x$ e um complemento las migas (as migalhas), que é o foco e que está co-indexado com o objeto do verbo comer. Enquanto a inserção como adjunto de $\mathrm{IP}_{\mathrm{COP}}$ fornece uma boa explicação para a proibição de movimento WH em (47b), (48) explica também porque o sujeito préverbal não pode ser focalizado enquanto o pós-verbal pode:

(49) a. *Fue JUAN comió las papas.

b. Comió papas fué JUAN.

(apud Camacho 2006)

O que a proposta não consegue explicar é a variação encontrada no espanhol caribenho quanto à aceitabilidade de focalizar o sujeito pós-verbal:

(50) a. *Canta muy bien es Juan.

b. *Fuma cigarillos Americanos es Juan. (apud Toribio 1992) 
A proposta em (48) parece, por fim, incapaz de derivar SCs em que o VP é focalizado.

Analisando sentenças do português brasileiro, Mioto 2008, 2012 propõe que a cópula é uma partícula focalizadora que, no domínio de c-comando de $\mathrm{T}$, se adjunge ao constituinte focalizado. Sua análise prediz que uma língua sem inversão livre, como o PB, dificilmente consegue focalizar o sujeito com uma SC, embora Wheeler 1982 apresente exemplos como o de (51b):

(51) a. *Fez a trapalhada foi o João. (apud Mioto 2008)

b. Me ajudou muito foi o psicólogo. (apud Weeler 1982)

O que nenhuma das análises brevemente apresentadas acima consegue derivar com naturalidade é o exemplo em (52) de Costa e Duarte (2003), com foco sobre os dois objetos:

(52) O João deu foi um kindle para a irmã mais nova.

$\mathrm{Na}$ tentativa de resolver também esse problema, fazemos a proposta abaixo.

\subsection{Nossa proposta}

Uma sentença como (53) tem a derivação em (54):

(53) O João comprou foi batata

(54) a. movimento do verbo para $\mathrm{T}$

$\left[_{T P}\right.$ O João comprou [ ${ }_{V P}$ eomprou batata] $]$

b. movimento do VP para FP na periferia esquerda da sentença (Rizzi 1997)

$\left[_{\mathrm{FP}}\left[{ }_{\mathrm{VP}}\right.\right.$ eomprou batata $\left[_{\mathrm{TP}}\right.$ o João comprou $\left.]\right]$

c. inserção da cópula

Foi $\left[_{\mathrm{FP}}\right.$ [eomprou batata] $\left[_{\mathrm{TP}}\right.$ o João comprou $]$

d. movimento remanescente do TP para posição de TopP (Kayne 2004)

$\left[_{\mathrm{TopP}}\left[_{\mathrm{TP}} \mathrm{O}\right.\right.$ João comprou] foi [ $\mathrm{VP}_{\mathrm{P}}$ emprot batata]]

Pressuposição foco

Observe agora as sentenças em (55):

(55) a. O João vai é dar um relógio para a mãe.

b. O João vai dar é um relógio para a mãe.

c. O João vai dar para a mãe é um relógio.

d. O João vai dar um relógio é para a mãe.

Tendo em mente que vai não é um afixo e que, por isso, o verbo não sobe para

$\mathrm{T}$, a derivação da sentença em (55a) é como (56): 
(56) a. Input

$\left[_{\mathrm{TP}} \mathrm{O}\right.$ João vai VPP $_{\mathrm{V}}$ dar um relógio para a mãe]]

b. movimento do VP para FP

$\left[_{\mathrm{FP}}\left[{ }_{\mathrm{VP}}\right.\right.$ dar um relógio para a mãe $\left[_{\mathrm{TP}}\right.$ o João vai]]

c. inserção da cópula

é [ ${ }_{F P}\left[\mathrm{VP}\right.$ dar um relógio para a mãe] $\left[_{\mathrm{TP}}\right.$ o João vai]]

d. Movimento remanescente de TP

[TopP $\left[_{T P}\right.$ o João vai] é [ ${ }_{V P}$ dar um relógio para a mãe]]

A derivação das outras sentenças de (55) apresentam algumas particularidades a mais. (55b) segue os mesmos passos de (56), mas tem um estágio a mais antes do movimento do VP: movimento do verbo para $v$. Em $(55 \mathrm{c})$, antes do movimento do VP para foco, movemos o V para $v$ e para a mãe para TopP na periferia do VP (Belletti 2004). Em (55d), movemos V para $v$ e um relógio para TopP na periferia do VP.

Segundo nossa proposta, a focalização é obtida via movimento do (que sobra do) VP para FP na periferia esquerda da sentença (Uriagereka 1995, Rizzi 1997). A cópula é um verbo impessoal sem traços EPP. O movimento para FP ou para TopP são do tipo "remanescente" (Kayne 2004).

Kato e Raposo 2007 mostram que, quando temos um tópico contrastivo, o que é movido para a periferia esquerda da sentença é o que sobrou do VP. Assim em (57a), não é apenas o objeto do verbo que é movido para a periferia esquerda da sentença, mas o VP remanescente:

(57) a. Inglês o Pedro fala, não o francês.

b. Falar inglês o Pedro fala, mas não o francês.

c. Fałar inglês o Pedro fala, mas não o francês.

Em nossa proposta, o que quer que sobre do VP pode ser focalizado: o VP inteiro se o verbo não sobe; quando o verbo sobe, os objetos e adjuntos. Note que nunca conseguimos focalizar o VP inteiro se o verbo tem que subir para $\mathrm{T}$, como mostra (58):

(58) *O João foi comprou batatas

Isso traduz formalmente a generalização de que SCs só focalizam constituintes no domínio de c-comando de T. Portanto, esse processo de focalização não alcança nem os sujeitos pré-verbais (c.f (49a) aqui repetido) nem os chamados advérbios altos:

(49) a. *Fue JUAN comió las

papas.

b. Comió papas fué JUAN. (apud Camacho 2006)

(59) a. *O João comprou batatas foi provavelmente.

b. *O João comprou batatas foi infelizmente. 
Admitindo que toda sentença tem um FP para que os traços de foco sejam checados, uma SC se distingue das outras por ter na numeração uma cópula. Como o foco que ela veicula é do tipo contrastivo, o (resto do) VP que se move deve alcançar a periferia esquerda da sentença (cf. Rizzi 1997). Uma PC se distingue de uma SC por ter, além da cópula, uma expressão WH que funciona como uma variável cujo valor é estabelecido pelo foco. A presença da variável WH é responsável pelas assimetrias entre PCs e SCs apontadas acima.

Buscando evidência em favor do movimento do TP remanescente, observamos que o japonês faz largo uso de sentenças como:

(60) [Yoku nemuru no] wa Fido-ga da.

[bem dormiu Comp $]_{\text {Top }}$ Fido $_{\text {Nom }}$ is

Matsuda 1996 as considera SCs e as analisa como contendo um operador nulo. $\mathrm{O}$ fato de a sequência entre colchetes ser marcada pela partícula de tópico /-wa/ pode ser tomado como evidência de que o movimento que está em jogo nas SCs é do tipo remanescente: TP se move para TopP.

\section{CONCLUSÕES}

O presente trabalho mostrou que:

(a) as SCs podem ser analisadas como construções independentes de PCs;

(b) as PCs são originariamente estruturas mono-clausais contendo um sintagma complexo contendo um XP e uma expressão WH que o redobra;

(c) na estrutura mono-clausal original se verificam as condições de localidade/ccomando necessárias para que as conexões relevantes sejam estabelecidas;

(d) as SCs só veiculam foco contrastivo/corretivo;

(e) o fato de as SCs só focalizarem elementos no domínio de c-comando de T deriva do movimento do VP residual;

(f) é o VP ou o que sobra dele que é movido para FP e não argumentos ou adjuntos;

(g) o que coloca a sentença na ordem final é o movimento residual do IP/TP para o TopP na periferia esquerda da sentença.

\section{REFERÊNCIAS BIBLIOGRÁFICAS}

AKMAJIAN, A. (1970). On deriving cleft sentences from pseudocleft sentence. Linguistic Inquiry 1: 149-168.

AMBAR, M. (2005). Clefts and tense asymmetries. In: Di Sciullo, A.M. (ed). UG and external systems. Amsterdam/USA: John Benjamins, p. 95-129.

BELLETTI, A. (2004). Aspects of the low IP area. In: Rizzi L. (ed. ). The Structure of CP and IP: the cartography of syntactic structures. Vol 2. Oxford/ New York: Oxford University Press, p. 16-51. 
BOECKX, C. (2007). Pseudoclefts: a fully derivational account. In: J. Bayer, T. Bhattacharya, M.T. Harry Babu (eds.) Linguistic theory and South Asian languages: essays in honour of K. A. Jayaseelan. Amsterdam/Philadelphia: John Benjamins, p. 29-40.

BOŠKOVIČ, Ž. (1997). Pseudoclefts. Studia Linguistica, 51, p. 235-277.

BOSQUE, I. (1999). On focus vs. wh-movement: the case of Caribbean Spanish. Sophia linguistica working papers in linguistics, p. 44-45.

CAMACHO, J. (2006). In situ focus in Caribbean Spanish: toward a unified account of focus. Procedings of the 9th Hispanic Linguistic symposium. Somerville, p. 1-11.

CHOMSKY, N. (1977). On WH movement. In: Culicover, R. P.; Wasow, T.; Akmajian, N, A. (eds). Formal syntax. New York: Academic Press, p. 71-132.

CHOMSKY, N. (1981). Lectures on government and binding. Dordrecht: Foris.

COSTA, J.; DUARTE, I. (2003). Minimizando a estrutura: uma análise unificada das construções de clivagem em português. Actas da APL, p. 627-638.

DEN DIKKEN, M. (2006). Relators and linkers: the syntax of predication, predicate inversion, and copulas. Cambridge, Mass.: The MIT Press.

HANKAMER, J. (1974). On the non-cyclic nature of Wh-clefting. Proccedings of CLS, 10. Chicago, IL: CLS. p. 221-233.

HIGGINS, F. R. (1973). The Pseudocleft Construction in English. MIT Ph.d. Dissertation.

KATO, M. A. (2009). VP-remnant movement in Portuguese focus constructions. Paper presented at THE $39^{\text {тн }}$ LSRL, Tucson. Arizona.

KATO, M. A. (2010). Clivadas sem operador no português brasileiro. Estudos da Lingua(gem). Vitoria da Conquista. 8:2. p. 61-77.

KATO, M. A.; Raposo, E. (2007). Topicalization in European and Brazilian Portuguese. In: J. Camacho, M.J. Cabrera, L. Sánchez e V.Deprez (eds). Romance Linguistics: selected papers from the 36th Linguistic Symposium on Romance Languages (LSRL). Amsterdam: John Benjamins. p. 213226.

KATO, M. A.; BRAGA, M. L.; CORREA, V.; LOPES-ROSSI, M.A.; SIKANSI, N. N. (1996). As construções-Q no português brasileiro falado. In: Koch, I.V. (ed). Gramática do português falado Vol VI. Campinas: Ed. Unicamp /FAPESP.

KATO, M. A.; MIOTO, C. (2011). Pseudo clivadas e os efeitos de conectividade. In: Estudos Formais da Gramática das Línguas Naturais - Anais do Encontro Nacional do Grupo de Trabalho Teoria da Gramática, p. 51-66. Goiânia: Editora Cânone.

KAYNE, R. (2002). Pronouns and their antecedents. In: S. Epstein and D. Seely (eds) Derivation and explanation in the minimalist program. Malden, Mass: Blackwell, p. 133-166.

KAYNE, R. (2004). The Antisymmetry of Syntax. Cambridge, Mass: The MIT Press.

MATSUDA, Y. (1996). A syntactic analysis of focus sentences in Japanese. MIT Working Papers in Linguistics 31, p. 291-310. 
MIOTO, C. (2008). Pseudo-clivadas reduzidas em espanhol caribenho e em português brasileiro. Paper presented at the III Workshop on Romania Nova. Montevideo.

MIOTO, C. (2012). Reduced pseudoclefts in Caribbean Spanish and in Brazilian Portuguese. In: Valentina Bianchi; Cristiano Chesi. (Orgs.). Enjoy Linguistics! Papers offered to Luigi Rizzi on the occasion of his 60th birthday. 1ed. Siena, IT: CISCL Press, p. 287-302.

MIOTO, C.; NEGRÃO, E. V. (2007). As sentenças clivadas não contêm uma relativa. In: A. de Castilho, M.A. Torres-Moraes, R.E.V. Lopes and S,M.L.Cyrino (eds) Descrição, História e Aquisição do Português Brasileiro. Campinas: Pontes, p. 159-183.

MODESTO, M. (1995). As construções clivadas no português do Brasil: relações entre interpretação focal, movimento sintático e prosódia. São Paulo: Humanitas, FLCH/USP.

RESENES, M. S. de. (2009). Sentenças pseudo-clivadas do português brasileiro. MA Thesis. UFSC, Florianópolis.

RESENES, M. S. de. (2014). A sintaxe das construções semiclivadas e pseudoclivadas do português brasileiro. f. 284. Tese (doutorado em linguística). São Paulo, USP.

RESENES, M.; DEN DIKKEN, M. (2012). Semi-clefts as a window on the syntax of predication, modification, and complementation. $48^{\text {th }}$ Annual meeting of the Chiago Linguistic Society. (CLS), Chicago.

RIZZI, L. (1997). The fine structure of the left periphery. In: L.M. Haegeman (ed.). Elements of grammar. Handbook of generative syntax. Dordrecht: Kluwer, p. 281-337.

TORÍBIO, A. J. (1992). Proper government in Spanish: subject relativization. Probus 4, p. 291-304.

URIAGEREKA, J. (1995). A F position in Western Romance. In: K. É. Kiss (ed.). Discourse configurational languages, p. 153-175.

WHEELER, D. (1982). Portuguese pseudoclefts: evidence for free relatives. Eighteenth Regional Meeting Chicago Linguistic Society, p. 507-520. 